\title{
COMPLETENESS OF HAMILTONIAN VECTOR FIELDS
}

\author{
DAVID G. EBIN
}

\begin{abstract}
We prove that under certain conditions the flow of a Hamiltonian vector field on a possibly infinite-dimensional dynamical system exists for all time.
\end{abstract}

We shall prove that under certain natural conditions the Hamiltonian vector field of a (possibly infinite-dimensional) dynamical system has integral curves which extend for all time. W. Gordon [3] has proved the result in the case of Euclidean space. We are grateful to J. Marsden for suggesting the problem and to W. Meyer for useful conversations.

Let $M$ be a smooth Hilbert manifold with a Riemannian structure, $\langle$,$\rangle , let V_{0}: M \rightarrow R$ be a smooth function and let $V=V_{0} \circ \pi$ where $\pi: T(M) \rightarrow M$ is the projection map of the tangent bundle. Define $H: T(M) \rightarrow R$ by $H=K+V$ where $K$, the kinetic energy, is defined by $K(V)=\frac{1}{2}\langle V, V\rangle$.

It is well known that $T^{*} M$ (and by means of $\langle\rangle,, T M$ ) has a natural symplectic two-form $\Omega$, and the equation $i(Z) \Omega=d H$ can be used to define a vector field $Z$ on $T M[4, \mathrm{p} .110]$.

THEOREM. If $M$ is complete under the metric induced by $\langle$,$\rangle and if$ $V$ is bounded below, then all integral curves of $Z$ extend for all time. Equivalently the flow $F: T M \times R \rightarrow T M$ which satisfies $(\partial F / \partial t)(X, s)$ $=Z_{F(X, s)}$ is defined on all of $T M \times R$.

To prove the theorem we must construct a Riemannian structure for $T M$ and this we do by use of the affine connection.

It is well known that $\langle$,$\rangle defines a unique connection and for$ each $p \in M$ and $X \in T_{p} M, \nabla$ defines a splitting of $T_{X} T M$ into vertical and horizontal subspaces; i.e. $T_{X} T M=V \oplus \mathcal{H C}$, [2]. Also there are canonical linear isomorphisms $L: \mathcal{V} \rightarrow T_{p} M$ and $T \pi: \Re \rightarrow T_{p} M, T \pi$ being the tangent map to $\pi$. We define an inner product, $\langle\langle\rangle$,$\rangle ,$ on $T_{X} T M$ by declaring $\mathcal{V}$ and $\mathfrak{F}$ perpendicular and $L$ and $T \pi$ isometries. This clearly defines a Riemannian structure for $T(M)$.

Lemma. $\langle\langle\rangle$,$\rangle induces a complete metric on TM.$

Received by the editors February 23, 1970.

AMS 1969 subject classifications. Primary 3465, 3480; Secondary 7040.

Key words and phrases. Hamiltonian vector field, Hilbert manifold, Riemannian structure, symplectic two-form, vertical subspace, horizontal subspace, spray, complete metric. 
Proof. Let $\left\{X_{i}\right\}$ be a Cauchy sequence in $T M$ and let $p_{i}=\pi(X i)$. For any $q \in M$ and $y \in T_{q} M, T \pi: T_{y} T M \rightarrow T_{q} M$ is distance decreasing. Hence $\pi$ decreases the length of curves, so $\left\{p_{i}\right\}$ is Cauchy in $M$.

Let $p=\lim _{i \rightarrow \infty}\left\{p_{i}\right\}$ and choose a closed neighborhood $U$ about $p$ and a chart $\varphi: U \rightarrow E$. ( $E$ is a Hilbert space on which $M$ is modeled.) $\varphi$ induces a chart $T \varphi: T(U) \rightarrow E \times E$ in a standard way [4, p. 40], and for $i$ sufficiently large $X_{i} \in T(U)$. We can assume that $\varphi(U)$ is a closed ball in $E$ in which case $T \varphi(T(U))=\varphi(U) \times E$ is a closed subset of $E \times E . \varphi(U) \times E$ is therefore complete as a metric subspace of $E \times E$, so if we can show $\left\{T \varphi\left(X_{i}\right)\right\}$ is Cauchy in $\varphi(U) \times E$ then $\left\{T \varphi\left(X_{i}\right)\right\}$ will converge and so will $\left\{X_{i}\right\}$.

To show $\left\{T \varphi\left(X_{i}\right)\right\}$ Cauchy, we make $\varphi: U \rightarrow E$ a normal coordinate chart so that $T T_{\varphi}: T_{y} T M \rightarrow U \times E \times E \times E$ is an isometry for all $y \in \pi^{-1}(p)$, and so that there exists a constant $A$ such that for any $W \in T T U,\|T T \varphi W\| \leqq A\|W\|$. The existence of such an $A$ means that all curves in $T U$ are stretched, under the map $T \varphi$, by at most a factor of $A$. Then, letting $\rho$ be the metric on $T M$, we must show that for $i, j$ sufficiently large and for any curve $\gamma$ of length sufficiently close to $\rho\left(X_{i}, X_{j}\right), \gamma$ is contained in $T U$. If this is true, $T \varphi(\gamma)$ has length at most $A$ times the length of $\gamma$ and the distance between $T \varphi\left(X_{i}\right)$ and $T \varphi\left(X_{j}\right)$ is at most $A \rho\left(X_{i}, X_{j}\right)$.

To show curves such as $\gamma$ are contained in $T(U)$ we assume $U$ is a metric ball about $p$ of radius $3 \epsilon$. Then let $V \subseteq U$ be the ball of radius $\epsilon$, and pick $i, j$ sufficiently large so that $\rho\left(X_{i}, X_{j}\right)<\epsilon$ and $X_{i}, X_{j} \in T(V)$. Then if $\gamma$ is a curve from $X_{i}$ to $X_{j}$ of length less than $2 \epsilon, \gamma_{0}=\pi \circ \gamma$ must also have length less than $2 \epsilon$ so $\gamma_{0} \subseteq U$. Thus $\gamma \leqq T U, T \varphi(\gamma)$ has length at most $2 A \epsilon$, and the distance from $T \varphi\left(X_{i}\right)$ to $T \varphi\left(X_{j}\right)$ is therefore at most $2 \epsilon$. Hence $\left\{T \varphi\left(X_{i}\right)\right\}$ is Cauchy and the lemma is proven.

Proof of Theorem. Consider the equation $i(Z) \Omega=d H$ and let $Z=S+G$ where $S$ satisfies $i(S) \Omega=d K$ and $G$ satisfies $i(G) \Omega=d V$. Then $S$ is the spray of the Riemannian metric $\langle\rangle,[4$, p. 110], and $G$ is the vertical lift of $\operatorname{grad} V$; i.e. $G_{x}=L^{-1}(\operatorname{grad} V)$ where $L: \mathcal{V}$ $\rightarrow T_{\pi(x)} M$. (See [1].)

Let $c:(a, b) \rightarrow T M$ be a maximal integral curve of $Z$. We wish to show that $a=-\infty, b=\infty$, and to do this, we first assume $a$ (respectively $b$ ) finite and show $\lim _{t \rightarrow a}\{c(t)\}$ (respectively $\lim _{t \rightarrow b} c(t)$ ) exists in TM. Then by the fundamental theorem of ordinary differential equations [4], the domain of $c$ extends to $(a+\epsilon, b)$ (respectively $(a, b+\epsilon)$ ). This contradicts the maximality of $(a, b)$ and proves the theorem.

The main fact needed to show $\lim _{t \rightarrow a}\{c(t)\}$ exists, is that $H$ is constant along $c$. This is simply conservation of energy and is proven 
in $\left[1\right.$, p. 110]. Let $N$ be the lower bound for $V$ and let $N_{1}=H(c)$. Then $K \uparrow c$ is bounded by $N_{1}-N$.

Let $c_{0}=\pi \circ c$, a curve in $M$. Then $c_{0}^{\prime}$, the tangent vector to $c_{0}$ satisfies $c_{0}^{\prime}(t)=T \pi\left(c^{\prime}(t)\right)=T \pi\left(Z_{c(t)}\right)$. But $T \pi\left(Z_{c(t)}\right)=T \pi\left(S_{c(t)}\right)=c(t)$ since $G$ is vertical and $S$ is a spray. Therefore since $K$ is bounded on $c$, the length of $c_{0}^{\prime}(t)$ is bounded and, since $M$ is complete, $\lim _{t \rightarrow b}\left\{c_{0}(t)\right\}$ exists. Let $c_{0}(b)=\lim _{t \rightarrow b}\left\{c_{0}(t)\right\}$.

Now we seek a limit of $c(t)$ in $\pi^{-1}\left(c_{0}(b)\right)$. To show that it exists we must only show that $c^{\prime}(t)$ is bounded in the metric on $T M$. $c^{\prime}(t)=Z_{c(t)}=S_{c(t)}+G_{c(t)}$, and $S$ and $G$ are horizontal and vertical respectively. Also $G_{c(t)}$ depends only on $c_{0}(t)$ and since $c_{0}(t)$ extends continuously to $c_{0}(b), G_{c(t)}$ remains bounded as $t \rightarrow b$. Also $\left\|S_{c(t)}\right\|$ $=\|c(t)\|$, from the definition of the metric on $T M$. It follows that $\left\|c^{\prime}(t)\right\|=\left\|S_{c(t)}\right\|+\left\|G_{c(t)}\right\|$ remains bounded as $t \rightarrow b$, so $c(t)$ converges. The same argument works as $t \rightarrow a$, and the theorem follows.

REMARK 1. If $M$ is finite-dimensional, one can use local compactness of TM instead of a metric on $T M$, so the proof is much shorter.

REMARK 2. It is easy to show that the theorem is false if $M$ is not complete or $V$ is not bounded below. There are counterexamples even if $M$ is one-dimensional.

\section{REFERENCES}

1. R. Abraham, Foundations of mechanics, Benjamin, New York, 1967. MR 36 \#3527.

2. P. Dombrowski, On the geometry of the tangent bundle, J. Reine Angew. Math. 210 (1962), 73-88. MR 25 \#4463.

3. W. Gordon, On completeness of vector fields (preprint).

4. S. Lang, Introduction to differentiable manifolds, Interscience, New York, 1962. MR 27 \#5192.

State University of New York, Stony Brook, New York 11970. 